\title{
Relative Stopping Power Measurements and Prosthesis artifacts reduction in proton CT
}

\author{
Carlo Civinini ${ }^{1}$, Monica Scaringella ${ }^{1}$, Mirko Brianzi ${ }^{1}$, Matteo Intravaia ${ }^{1,2, *}$, Nunzio \\ Randazzo $^{3}$, Valeria Sipala ${ }^{3,4}$, Marta Rovituso ${ }^{5,+}$, Francesco Tommasino ${ }^{5,6}$, Marco \\ Schwarz ${ }^{5,7}$ and Mara Bruzzi ${ }^{1,8}$ \\ ${ }^{1}$ Istituto Nazionale di Fisica Nucleare sezione di Firenze, Via G. Sansone 1, Sesto Fiorentino (Fi), Italy \\ ${ }^{2}$ Dipartimento di Scienze dell'Informazione Università degli Studi di Firenze, Via S.Marta 3, Firenze, \\ Italy \\ ${ }^{3}$ Istituto Nazionale di Fisica Nucleare sezione di Catania, Via S. Sofia 64, Catania, Italy \\ ${ }^{4}$ Dipartimento di Chimica e Farmacia Università degli Studi di Sassari, via Vienna 2, Sassari, Italy \\ ${ }^{5}$ Istituto Nazionale di Fisica Nucleare TIFPA, via Sommarive, 14, Povo (Tn), Italy \\ ${ }^{6}$ Dipartimento di Fisica Università di Trento, via Sommarive 14, Povo (Tn), Italy \\ ${ }^{7}$ Agenzia Provinciale per i Servizi Sanitari, Via Al Desert 14, (Tn), Italy \\ ${ }^{8}$ Dipartimento di Fisica e Astronomia, Università degli Studi di Firenze, via G. Sansone 1, Sesto \\ Fiorentino (Fi), Italy \\ E-mail: carlo.civinini@fi.infn.it
}

Received xxxxxx

Accepted for publication $\mathrm{xxxxxx}$

Published xxxxxx

\begin{abstract}
We present a set-up for proton Computed Tomography (pCT), composed of a microstrip silicon tracker and a YAG:Ce calorimeter, able to directly measure the Relative Stopping Power (RSP) maps to be used in hadron therapy. The system, tested with an electron density calibration phantom at the Trento proton Therapy Center, is able to correlate measured and expected RSP with discrepancies less than 1\%. Further, proton-CT tomographies of an anthropomorphous head phantom taken with our device, when compared with $\mathrm{x}-\mathrm{CT}$ images of the same object, evidence a significant reduction of artifacts induced by titanium spinal bone prosthesis and tungsten dental filling.
\end{abstract}

Keywords: Hadron Therapy, Proton Tomography, Stopping Power, Algebraic Reconstruction Techniques, Metallic Prosthesis

\section{Introduction}

Hadron therapy irradiates the tumor volume voxel by voxel by moving the proton / ion beam and modulating the particles energy to correctly place the Bragg's peak within the selected position, while sparing as much as possible the surrounding healthy tissues. To this purpose, Treatment Planning Systems calculate the particle kinetic energy of the therapeutic beam and its direction using the proton stopping power normalized to liquid water (RSP) 3D distribution in the region crossed by the beam. Presently the RSP maps, are extracted from conventional $\mathrm{x}$-rays $\mathrm{CT}(\mathrm{x}-\mathrm{CT})$ images translating the photon attenuation coefficients (Hounsfield's Units - HU) by appropriate conversion and calibration coefficients [1]. This method introduces an uncertainty on the position of the Bragg's peak in the patient which may significantly reduce the

\footnotetext{
${ }^{*}$ Current address: Dipartimento di Ingegneria dell’Informazione e Scienze Matematiche, Università di Siena, Italy

$\dagger$ Current address: Holland proton therapy center (HollandPTC), Delft, Holland
} 
advantages offered by the high spatial selectivity of hadrons. It is common practice in hadron therapy to assume an uncertainty of about 3\% in the estimated range, and to compensate for that in the planning phase via intended "overshooting", thus leading to an increased volume of healthy tissue being irradiated [2]. A 'proton Computed Tomography' (pCT) system, which directly measures the 3D RSP maps using the same irradiation beam with a much reduced intensity $\left(\sim 10^{6}\right.$ particles/s) and a sufficient kinetic energy to be used in "transmission mode", can mitigate this source of error. Further, pCT images can be of paramount importance when the therapeutic beam crosses regions where metal prosthesis are implanted. In fact, high Z materials are responsible of the appearance of severe artifacts in the $\mathrm{x}-\mathrm{CT}$ images [3]. In such regions the estimation of the RSP maps becomes difficult due to the mismeasurement of the HU values. An extensive review of the potential advantages of $\mathrm{pCT}$ techniques for increasing accuracy in proton therapy can be found in [4].

In the present work, we describe the results obtained with a pre-clinical $\mathrm{pCT}$ system quantifying these advantages in terms of accuracy in RSP measurement and artifact reduction.

Our pCT system is based on a silicon microstrip tracker and a Cerium-doped Yttrium Aluminum Garnet (YAG:Ce) scintillating calorimeter, with a $5 \times 20 \mathrm{~cm}^{2}$ field of view. It has been developed starting from a former prototype of smaller active area [5-12]. We present results of the experimental tests carried out at the Trento Proton Therapy Center (APSS, Azienda Provinciale per i Servizi Sanitari, Trento, Italy) with this device and two test objects (phantoms). One of these phantom, used for electron density calibration, shows that pCT can indeed obtain a reliable correlation between expected and measured RSPs. The other, an anthropomorphous head phantom, is used to investigate the potential of pCT in improving tomographic imaging as a consequence of the reduced impact of the artifacts induced by metallic prosthesis, against $\mathrm{x}-\mathrm{CT}$.

\section{Experimental Procedures}

The main difficulty encountered to increase accuracy with pCT is Multiple Coulomb Scattering (MCS). In fact, with typical targets as thick as $20 \mathrm{~cm}$ (water equivalent), a $200 \mathrm{MeV}$ proton beam undergoes an rms MCS angle of about $30 \mathrm{mrad}$.

On a $10 \mathrm{~cm}$-distant plane, this brings the projected displacement distribution up to a $3 \mathrm{~mm}$, or even higher, rms. Therefore, simple proton projection radiographies cannot be used to extract the RSP map with a good precision.

The pCT solution to this problem is based on reconstructing the most likely path (MLP) of each proton crossing the object under test. In a $\mathrm{pCT}$ apparatus, the MLP is determined by sampling each proton trajectory both up- and down-stream the target, thus reconstructing two segments [13]. In this way, the maximum value of the one sigma error envelope on a transverse coordinate of the MLP of, e.g., a $200 \mathrm{MeV}$ energy proton in $20 \mathrm{~cm}$ of water, is about $0.5 \mathrm{~mm}$ [14]. Each singleevent information in a pCT is composed of the proton MLP estimated using the measurement coming from the tracker, and the proton energy loss measured by the calorimeter. A complete data-set needs measurements taken by rotating the phantom at many different angles. Then, Algebraic Reconstruction Techniques (ART) are used to reconstruct the 3D RSP maps [15]. In the forthcoming sections of this chapter we will discuss in detail the main characteristics of our pCT system, the phantom and the proton beam used.

\section{1 pCT Apparatus}

Figure 1 shows a picture of the complete apparatus mounted on the 'Trento Proton Therapy Center' experimental beam line.

The tracker is composed of four planes, each able to measure the 3D coordinate of the proton impact point to be used by the MLP algorithm to reconstruct the proton trajectory. A tracker plane is made by a single printed circuit board hosting the silicon microstrip sensors, the front-end and the digital read-out electronics. The silicon microstrip sensors are glued onto a rectangular aperture $\left(20.3 \times 4.9 \mathrm{~cm}^{2}\right)$ at the center of the board: four with the strips vertical, measuring the $\mathrm{x}$ coordinate ( $\mathrm{x}$-side) and four with strips horizontal, measuring the y coordinate (y-side). The two units are glued on the two sides of the board with the sensors mounted one next to the other with a small overlap to guarantee hermeticity. Each strip of an $\mathrm{x}$-side sensor is connected to a front-end chip channel while two strips of the y-side sensors are daisy chained and readout by a single channel. In that way four groups, made up of eight front-end chips each, readout the $\mathrm{x}$ side while two groups are used for the y-side. The strips are connected to the front-end electronics through a pitch-adapter directly designed on the printed circuit board.

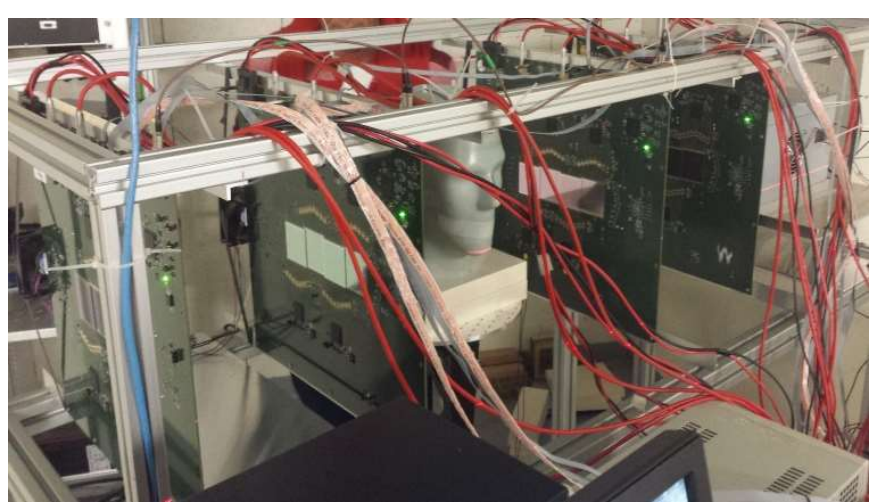

Fig. 1. Picture of our pCT apparatus installed at the 'Trento Proton Therapy Center' experimental beam line. The proton beam enters the apparatus from the left. The tracker planes (green boards) and the calorimeter (grey box) are visible together with the anthropomorphous head phantom. 
The single-sided silicon microstrip sensors are of p-on-n type, with 256 strips at a pitch of $200 \mu \mathrm{m}$, a $5.1 \times 5.1 \mathrm{~cm}^{2}$ sensitive area and a thickness of $320 \mu \mathrm{m}$. The sensors have full depletion voltages around $85 \mathrm{~V}$ and an average bias current density at depletion of $2.5 \mathrm{nA} \mathrm{cm}^{-2}$. The tracker front-end ASIC [16] is a VLSI device, developed by our collaboration, consisting of 32-channels, and equipped with a charge amplifier, a shaper and a comparator producing a binary output by comparison with a threshold. They are connected to the data acquisition control system through an I2C line used to download the threshold values of each channel. The 32 digital output lines of the chip are connected to an FPGA (Xilinx, Spartan 6) which serves the group of 8 chips connected to a sensor. The six FPGA of a tracker plane (4 on the $\mathrm{x}$-side and 2 on the $\mathrm{y}$-side) are programmed to continuously sample the digital inputs from the front-end chips at $50 \mathrm{MHz}$ keeping the information on a 128 cells deep circular buffer. After receiving a trigger signal from the calorimeter electronics the FPGA scans in parallel the 256 inputs, using also pre-trigger samples, to find active strips. Only for them, information concerning initial time with respect to the trigger, signal duration and strip number are assembled within the six first level FPGA and then moved to a second level FPGA on the same plane, using 8 lines buses running at an aggregate $1.6 \mathrm{~Gb} \mathrm{~s}^{-1}$ transfer rate. The second level FPGA, after event synchronism control, assembles the fired strips in each of the six groups attaching to the data structure the event number and its time. After data building, the information is transferred to a third level FPGA (Virtex 6, Xilinx), for the final event assembly of the four tracker planes together with the calorimeter data.

The pCT apparatus is completed by a YAG:Ce calorimeter made by a $2 \times 7$ scintillating crystal matrix, placed just after the fourth tracker plane. Each crystal has a $3 \times 3 \mathrm{~cm}^{2}$ section and a $10 \mathrm{~cm}$ length and is readout by a silicon photodiode $(1.8 \mathrm{x}$ $1.8 \mathrm{~cm}^{2}$ area) which is insensitive to the stray magnetic field commonly present at the end of a transmission beam line. The YAG:Ce crystal has been selected both for its short light decay constant (70 ns) and an emitting light spectrum well matching the photodiode sensitivity range. The 14 analog signals are amplified, shaped and then digitally converted at $5 \mathrm{MHz}$ and 14 bits. Each event contains 32 samples per crystal and it is transferred to the main DAQ unit.

\subsection{Test Phantoms}

Figure 2 (a) shows the phantom used for electron density calibration (model CIRS 062M); this is an $18 \mathrm{~cm}$ diameter cylinder, $5 \mathrm{~cm}$ height, made of water equivalent plastic (Plastic Water $\left.{ }^{\circledR}-\mathrm{LR}\right)$. The phantom contains nine exchangeable inserts made of different tissue substitutes (see table I).

The central insert contains a vial filled with distilled water, used as reference to compute the RPS of the various materials.
TABLE I

ELECTRON DENSITY PHANTOM (CIRS 062M) MATERIAL DENSITY AND EXPECTED SP AND RSP VALUES FOR $180 \mathrm{MEV} \mathrm{PROTONS}$

\begin{tabular}{lcll}
\multicolumn{4}{c}{ EXPECTED SP AND RSP VALUES FOR 180 MEV PROTONS } \\
\hline \hline \multicolumn{1}{c}{ Material } & $\begin{array}{l}\text { Density } \\
\mathrm{g} / \mathrm{cm}^{3}\end{array}$ & $\begin{array}{l}\text { Expected SP } \\
\mathrm{MeV} / \mathrm{cm}\end{array}$ & Expected RSP \\
\hline Lung (inhale) & 0.205 & 0.979 & 0.204 \\
Lung (exhale) & 0.507 & 2.449 & 0.511 \\
Adipose & 0.96 & 4.655 & 0.971 \\
Breast & 0.99 & 4.775 & 0.996 \\
Distilled Water & 1 & 4.792 & 1 \\
Plastic equiv. water & 1.029 & 4.822 & 1.006 \\
Muscle & 1.06 & 5.076 & 1.059 \\
Liver & 1.07 & 5.133 & 1.071 \\
Bone (trabecular) & 1.16 & 5.357 & 1.118 \\
Bone (dense) & 1.53 & 6.767 & 1.412
\end{tabular}

The expected SP and RSP in table I are computed using Geant4 [17-19], starting from the elemental inserts' composition [20] and density.

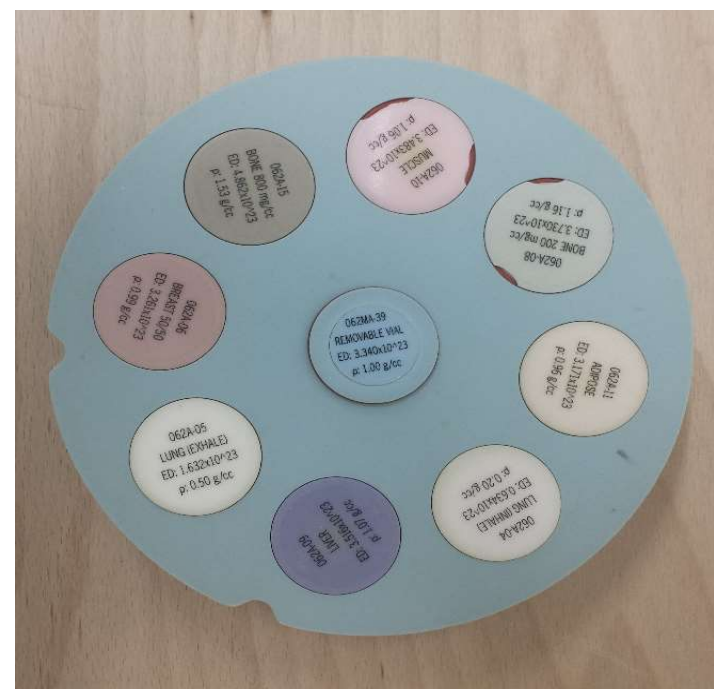

Fig. 2 (a) Picture of the electron density calibration phantom with nine inserts of different tissue equivalent materials (see table I).

We also studied an anthropomorphous average male head phantom (CIRS 731-HN) manufactured with tissue-equivalent materials, which mimic reference tissues within $1.5 \%$ for protons. Figure 2 (b) shows its x-ray radiography.

Internal structures of the phantom include brain, bone with cortical and trabecular distinction, larynx, trachea, fully-open sinus cavities, nasal and mouth cavities, and teeth with distinct dentine, enamel and root structure. 


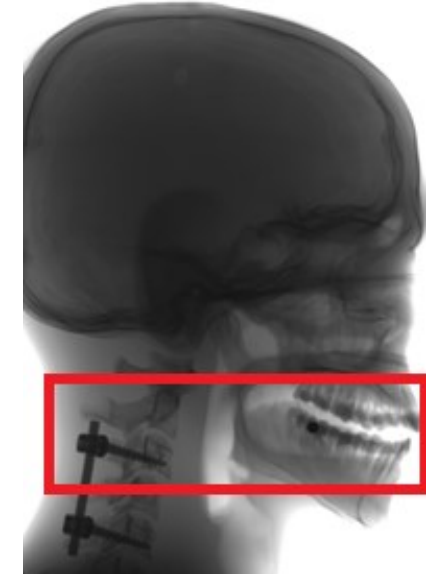

Fig. 2 (b) X-ray radiography of an anthropomorphic average male head phantom: a tungsten dental filling and a titanium vertebra prosthesis are visible. The red box highlights the volume where the proton tomography has been performed.

Furthermore, a tungsten filling in a molar and a titanium prosthesis attached by two screws at $\mathrm{C} 3$ and $\mathrm{C} 5$ vertebra are present. These metal inserts are useful to study the possible artifacts generated by high density materials. The phantom is composed by four vertical sections with narrow gaps between them to allow the insertion of radiochromic films.

\subsection{Experimental Proton Beam Line}

The Trento Proton Therapy Center experimental beam line has been extensively characterized in [21]. The pCT apparatus has been installed with the first tracker plane at a distance of $363 \mathrm{~cm}$ from the beam pipe vacuum exit window. The distance between the first and the second tracker plane, as well as the one from the third and fourth, has been set to $30 \mathrm{~cm}$. The space left free between the second and third plane, where the phantoms have been installed, was $50 \mathrm{~cm}$.

A nominal proton kinetic energy of $211.2 \mathrm{MeV}$ has been used during the full tomographic data taking. This corresponds to a measured proton energy at the iso-center $(125 \mathrm{~cm}$ from the beam pipe exit window) of $210 \mathrm{MeV}$.

A $2.5 \mathrm{~cm}$ thick tantalum plate has been placed just after the pipe end to spread the beam in order to cover the full pCT field of view. The average proton energy loss inside the plate has been taken into account to evaluate the correct energy loss inside the phantom.

\section{Image Reconstruction Methods}

\subsection{Tomographic Reconstruction Equations}

To reconstruct tomographic images of the phantom SP an ART iterative algorithm [22] has been implemented. The proton SP map, $S(x, y, E)$, is defined as:

$$
-d E=S(x, y, E) d l
$$

where $-d E$ is the energy loss of a particle with energy $E$ in a $d l$ path at point $x, y$. Introducing the mass stopping power as $\frac{S}{\rho}(x, y, E)=\frac{S(x, y, E)}{\rho(x, y)}$, (1) can be rewritten as:

$$
-\frac{s}{\rho}\left(x, y, E_{0}\right) d E=\frac{s}{\rho}\left(x, y, E_{0}\right) \frac{s}{\rho}(x, y, E) \rho(x, y) d l
$$

Dividing (2) by the mass stopping power at energy $E$ :

$-\frac{\frac{S}{\rho}\left(x, y, E_{0}\right)}{\frac{S}{\rho}(x, y, E)} d E=S\left(x, y, E_{0}\right) d l$,

$E_{0}$ being a reference energy at which the stopping power is calculated (e.g., $180 \mathrm{MeV}$ for this analysis). At this energy the stopping power ratio of (3) does not depend too much $(<6 \%)$ on the material composition and could be replaced by the one measured for liquid water [23]:

$$
-\left[\frac{S}{\rho}\left(H_{2} O\right)\right]_{E}^{E_{0}} d E=S\left(x, y, E_{0}\right) d l
$$

where:

$$
\left[\frac{S}{\rho}\left(H_{2} O\right)\right]_{E}^{E_{0}} \cong\left[\frac{S}{\rho}(x, y)\right]_{E}^{E_{0}}=\frac{S}{\rho}\left(x, y, E_{0}\right) / \frac{S}{\rho}(x, y, E)
$$

Integrating along the proton path we obtain the tomographic equation:

$$
-\int_{E_{\text {in }}}^{E_{\text {out }}}\left[\frac{S}{\rho}\left(H_{2} O\right)\right]_{E}^{E_{0}} d E=\int_{\text {path }} S\left(x, y, E_{0}\right) d l
$$

In (6), $E_{\text {in }}$ and $E_{\text {out }}$ are the proton energies entering and exiting the phantom; 'path' is the better estimation of the proton trajectory inside the phantom as extracted using the MLP formalism $[13,14]$ from the tracker measurements.

A discretization of (6) can be done by dividing the phantom into a set of $N$ pixels. For $M$ protons the following set of linear equations can be written:

$$
\begin{array}{r}
p_{i} \equiv-\int_{E_{\text {in }}}^{E_{\text {out }}}\left[\frac{S}{\rho}\left(H_{2} O\right)\right]_{E}^{E_{0}} d E=\sum_{j=1}^{N} w_{i j} S_{j}\left(E_{0}\right) \\
i=1, \ldots, M
\end{array}
$$

where $p_{i}$ is measured using the calorimeter information for $i$-th proton and $w_{i j}$ is the trajectory length of $i$-th proton in the pixel $j$. The tomographic reconstruction problem [24] is then reduced to solve for $S_{j}\left(E_{0}\right)$, the stopping power value at pixel $j$ for energy $E_{0}$, the linear system (7).

\subsection{Iterative Algebraic Reconstruction Algorithms}

Large linear equation systems like (7) are usually solved by means of algebraic techniques [22]. These methods start from an arbitrary initial image, which could be a totally 'blank' picture or an approximation of the phantom obtained with less 
precise but faster methods, and iteratively project this image onto each of the equation (hyperplanes) (7) obtaining a sequence of points in the unknown $N$-dimensional space which converge to the solution.

The system (7) typically has more equations than unknowns. This, together with the uncertainties introduced by the finite apparatus resolutions, implies that an image which exactly satisfies all conditions (7) doesn't exist. Nonetheless, the sequence obtained iteratively projecting the image onto the hyperplanes, converges to an $\mathrm{N}$-dimensional volume where the hyperplane intersection density is high and not too far from the real image.

The ART iterative formula used to solve (7) is defined as:

$$
S^{k+1}=S^{k}+\lambda_{k} \frac{p_{i(k)}-\left\langle w^{i(k)} \mid S^{k}\right\rangle}{\left\|w^{i(k)}\right\|^{2}} w^{i(k)}
$$

where $i(k)=k \bmod M+1$ is a cyclic sequence on the event number (e.g., for 3 events $i(k) \rightarrow 1,2,3,1,2,3, \ldots$ ), $S^{k}$ is the $N$-dimensional image vector at iteration $k, w^{i(k)}$ the $i(k)^{\text {th }}$ track length in each pixel and $p_{i(k)}$ is the stopping power integral of the $i(k)^{\text {th }}$ proton (7). The relaxing factor $\lambda_{k}$ could be a constant or a sequence converging to zero. The algorithm starts with an image $S_{0}$ and stops if the quantity $\Delta=\left\|S^{i(k+1)}-S^{i(k)}\right\|$ is below a threshold. In (8) $\langle a \mid b\rangle$ is the scalar product of the two vectors, while $\|a\|$ is the Euclidean norm.

To reduce the 'salt-pepper' noise introduced by the resolution-induced internal inconsistency of (7), a variation of ART has been introduced. This technique (Simultaneous Algebraic Reconstruction Technique, SART [25]) still uses the projection of the image as in (8) but updates $S^{k}$ using an average over the full data set of the projections (9):

$$
S^{k+1}=S^{k}+\frac{\lambda_{k}}{M} \sum_{i=1}^{M} \frac{p_{i}-\left\langle w^{i} \mid S^{k}\right\rangle}{\left\|w^{i}\right\|^{2}} w^{i}
$$

SART has a fundamental computational advantage with respect to ART: since the correction to the image is done at the end of a cycle over the total number of events, it is easily parallelizable. A drawback is that the average could be a small number ( $M$ is large), so increasing the convergence time.

To overcome this problem, the data set is divided into a number of blocks and the average is not done on the event number but on the number of tracks intercepting a particular pixel [26]:

$$
S_{j}^{k+1}=S_{j}^{k}+\frac{\lambda_{k}}{T_{j}^{t(k)}} \sum_{i \in B_{t(k)}} \frac{p_{i}-\left\langle w^{i} \mid S^{k}\right\rangle}{\left\|w^{i}\right\|^{2}} w_{j}^{i} .
$$

In (10), the total data set $I$ has been divided into $T$ blocks $B_{t}$ such that $I=\bigcup_{t=1}^{T} B_{t}$, the iterations are done over a cyclic sequence of blocks $t(k)=k \bmod T+1, T_{j}^{t(k)}$ being the number of tracks in block $B_{t(k)}$ intersecting the pixel $j$.

\section{Results}

\subsection{Electron Density Calibration Phantom}

The tomographic reconstruction of the electron density calibration (EDC) phantom has been done in a cylindrical volume with the axis coincident with the rotational one. The volume has been subdivided into elementary voxels $0.600 \times 0.600 \times 1.650 \mathrm{~mm}^{3}$ each: the $600 \mu \mathrm{m}$ sizes are within the plane orthogonal to axis, while the latter one is the tomography slice thickness. The total number of voxels is $512 \times 512 \times 32$, namely $8.39 \times 10^{6}$.

Figure 3 shows the central slice of the reconstructed image. The image clearly shows the presence of all the different material inserts, besides the two indents at perimeter. Indeed, we can recognize the presence of air gaps around some of the inserts (breast, liquid water and adipose). As expected, the higher contrast is obtained for lung and bone which are characterized by a SP quite different from that of the plastic equivalent water matrix (see table I).

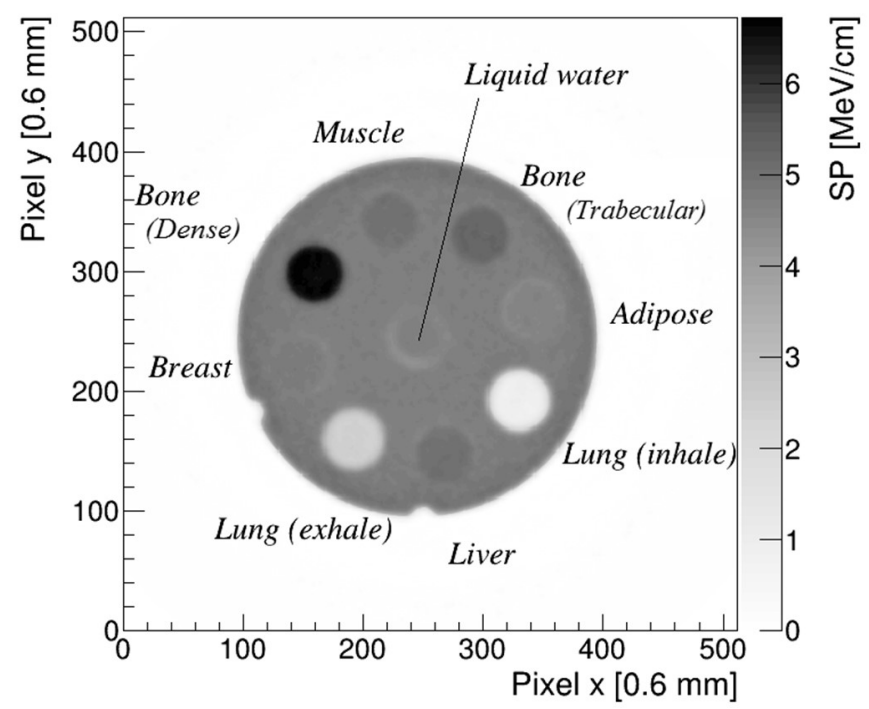

Fig. 3 The central slice $(1.65 \mathrm{~mm}$ thick) reconstructed image of the EDC phantom SP tomography. The grey levels are proportional to the absolute SP.

To quantify the ability of the pCT system to reconstruct the correct RSP values, we have considered fiducial volumes inside the various inserts and the plastic water bulk. Then, we evaluated the RSP distributions, defined as the SP divided by the average of the distilled water one. As an example, figures 4 (a) and (b) show the RSP of distilled water and plastic water distributions, respectively. Results have been fitted with a Gaussian to get mean and sigma values, to be compared with the expected ones reported in table I. The measured RSP of 
the Plastic Water is 1.006 as expected. Standard deviations of liquid and plastic water RSP distributions are similar $\sigma=$ $0.6 \%$. The same evaluation has been done for all the other inserts.
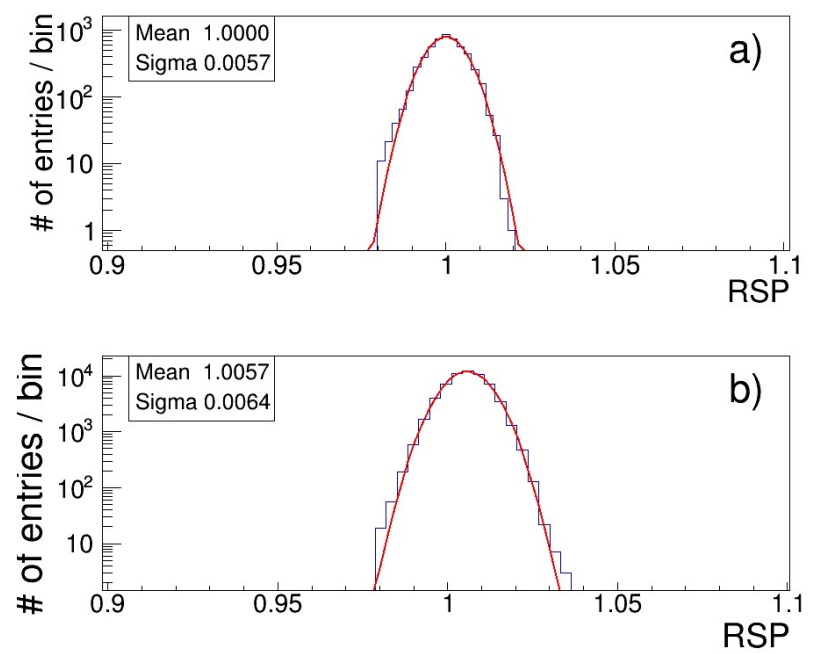

Fig. 4 RSP distributions for a) liquid and b) plastic water. Both distributions are fitted to a Gaussian function to get mean and sigma values, reported in the insets.

Figure 5 shows the correlation between measured and expected RSPs. Here the systematic error on the expected values has been assumed to be the difference between the predicted value for liquid water and the corresponding one obtained from the NIST table [23]. The error on the measured RSP is the standard deviation calculated with the Gaussian fit. The inset in the figure 5 is a detailed view of the region closest to the water RSP value, corresponding to the soft-tissue range.

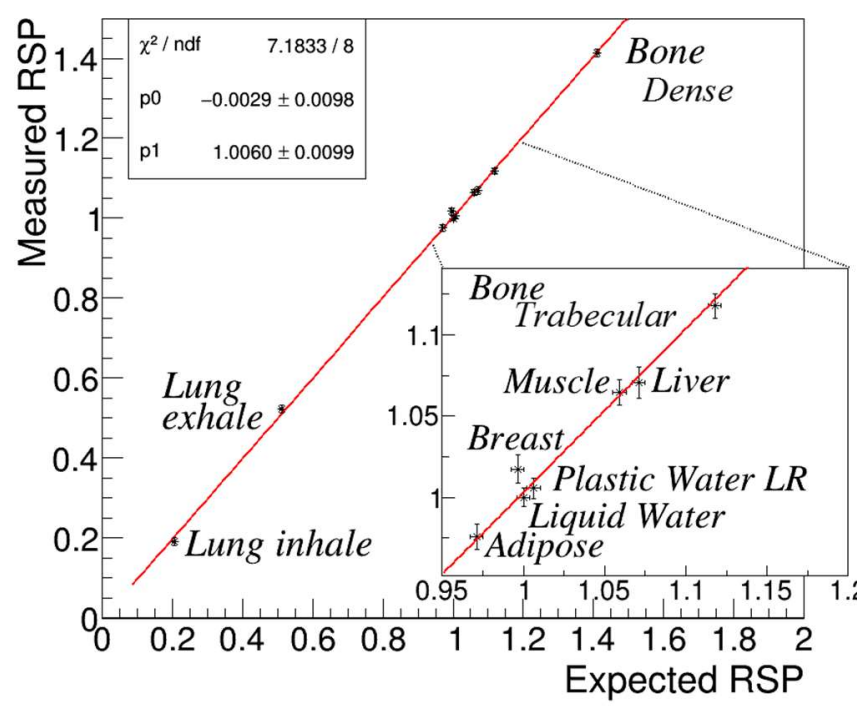

Fig. 5 Comparison between measured and expected RSP values of the certified materials within the EDC-phantom. Data fitted to a linear function. Inset: detailed view of the region closest to $\mathrm{RSP}=1$.
Data have been fitted with a linear regression: $y=p_{1} x+p_{0}$. A very good correlation is evidenced by the slope and intercept values, compatible respectively with 1 and 0 within their uncertainties. The quality of the fit is excellent, as evidenced by the $\chi^{2}$ value.

\subsection{Anthropomorphous Head Phantom}

The pCT image of the anthropomorphous head phantom has been reconstructed in a volume identical to the one used for the EDC phantom, where the vertical number of slices have been doubled halving the voxel volume $\left(0.600 \times 0.600 \times 0.825 \mathrm{~mm}^{3}\right)$. As a consequence, the total number of voxels has been doubled $(512 \times 512 \times 64)$. All the following images have been processed using ImageJ [27] starting from the reconstructed RSP values.

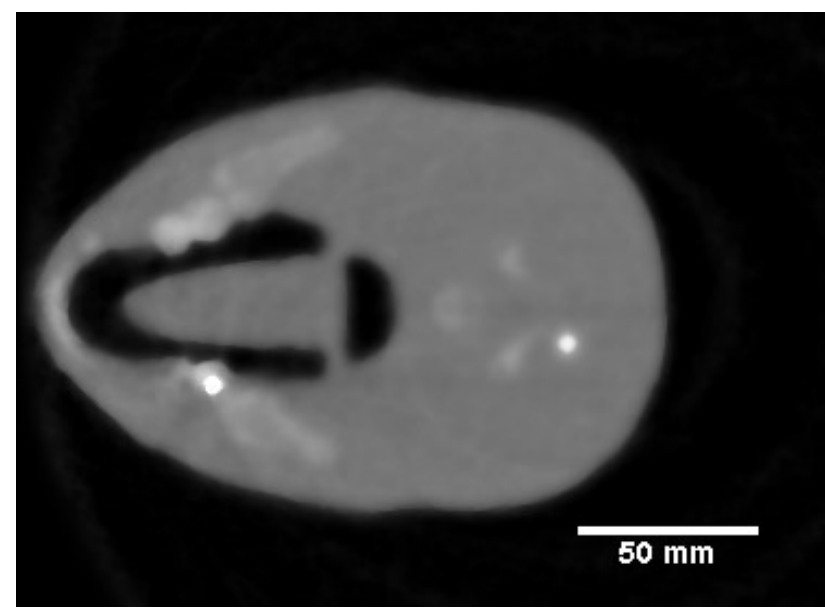

(a)

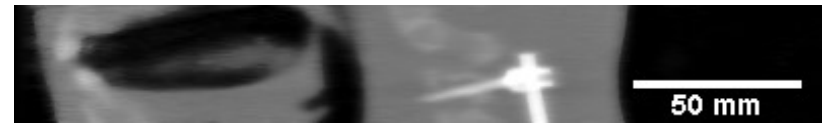

(b)

Fig. 6 Anthropomorphic head phantom pCT image: (a) horizontal slice of the dental filling region. (b) Vertical slice in a plane containing the titanium vertebral prosthesis.

Figure 6 (a) shows a pCT horizontal slice of the phantom at the level of the tungsten dental filling. Figure 6 (b) shows a pCT vertical slice of the phantom in a plane which contains the titanium prosthesis. As it can be seen, the anatomical details are clearly visible and no artifacts are present in both images. We can clearly distinguish the larynx and the mouth empty spaces together with the tongue and the lower dental arc. Two bright spots are visible in figure 6 (a): the tungsten dental filling and a section of the titanium vertebra prosthesis. This latter is visible, close to the spine, in figure 6 (b). Here, we can also clearly distinguish the screw inserted into the 
vertebra. In figure 6 (a) we can also distinguish the three narrow gaps for radiochromic film insertion.

\section{Metal Artifacts Analysis}

Results described in the previous section show that our pCT system is able to get a very accurate and direct measurement of the RSP values of (surrogate) biological tissues. This is in itself an outcome putting into evidence the importance of $\mathrm{pCT}$ as a tool to significantly improve the accuracy in hadron therapy treatments against the standard $\mathrm{x}-\mathrm{CT}$.

Going further, in this work we investigated the effect of metallic prosthesis on the image quality of pCT vs $\mathrm{x}-\mathrm{CT}$. To this purpose, figure 7 shows the $\mathrm{x}-\mathrm{CT}$ image of the anthropomorphous head phantom shown in figure 6 (a) (same slice). Here the voxel volume is $0.809 \times 0.809 \times 1.230 \mathrm{~mm}^{3}$.

Evident artifacts produced in the $\mathrm{x}-\mathrm{CT}$ image by the high density / high $Z$ values of the tungsten and titanium materials are clearly visible. Conversely, in figure 6 (a) the metal induced artifacts are much less evident. Nonetheless, the image here appears much more blurred, due to the lower spatial resolution of $\mathrm{pCT}$, an obvious effect of MCS, so it is not straightforward to draw stronger conclusions from this qualitative evidence.

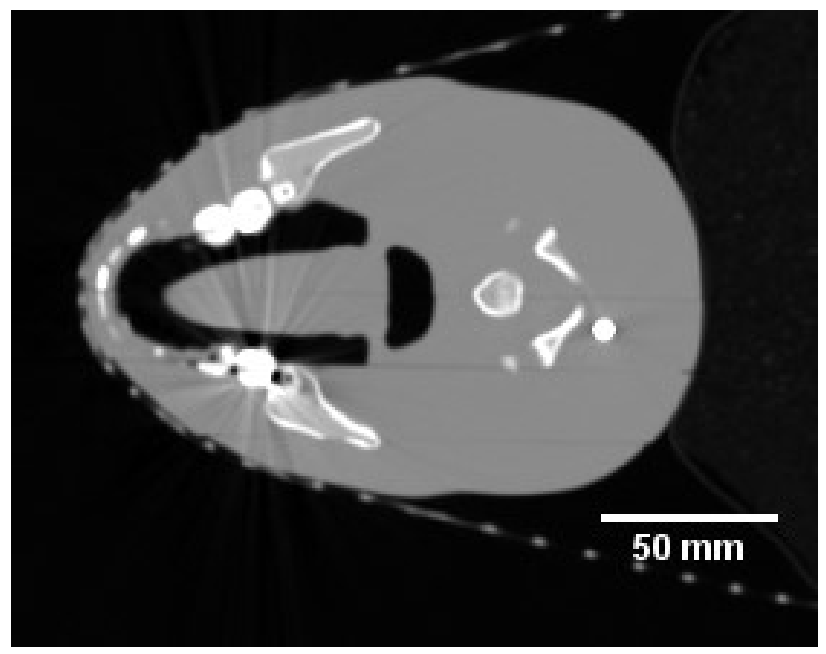

Fig. 7 Horizontal slice of the anthropomorphous phantom head x-CT tomography.

To visually enhance and compare the two images in the regions closest to the metal inserts, we show, in figure 8 (a) and (b) respectively for the $\mathrm{x}-\mathrm{CT}$ and $\mathrm{pCT}$ horizontal slices, zoomed regions close to the dental tungsten filling; same for titanium prosthesis is shown in figures 9 (a) and (b).

Here the full grey scale corresponds to a same $40 \%$ range around distilled water value $(800 \leq \mathrm{HU} \leq 1200$ and $0.8 \leq \mathrm{RSP}$ $\leq 1.2$ ). Figures 8 (a) and 9 (a) clearly show artifacts induced by the prosthesis in $\mathrm{x}-\mathrm{CT}$. These artifacts appear much attenuated in pCT images of figures 8 (b), 9 (b).

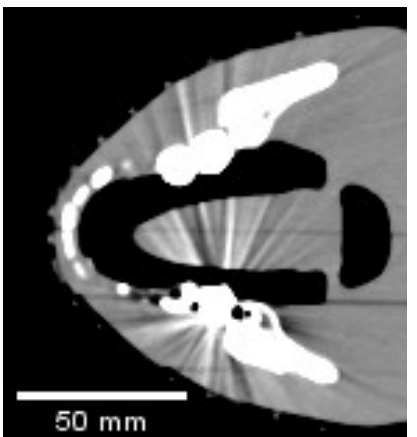

(a)

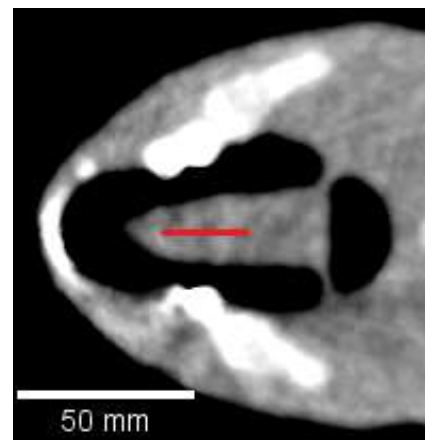

(b)
Fig. 8 Close-up view, with enhanced contrast, of the tungsten dental filling region: (a) X-CT shown in figure 7; (b) pCT shown in figure 6 (a). Red line indicates the region of interest (ROI) used to quantify the standard deviation of the HU and RSP values.

A quantitative comparison between pCT and $\mathrm{x}-\mathrm{CT}$ tomographic images in the presence of the two metallic objects has been done by calculating the standard deviation of the RSP and HU values in selected regions of interest (ROI) close to tungsten and titanium prosthesis, where the material is expected to be uniform.

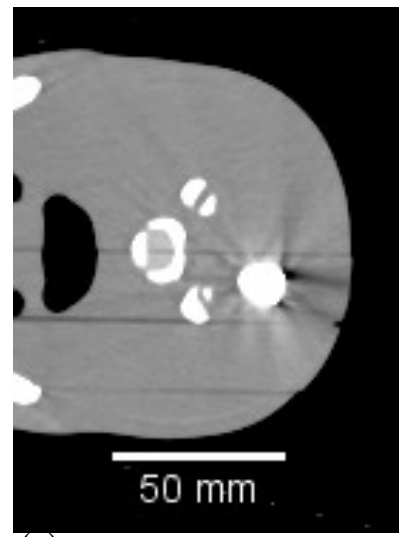

(a)

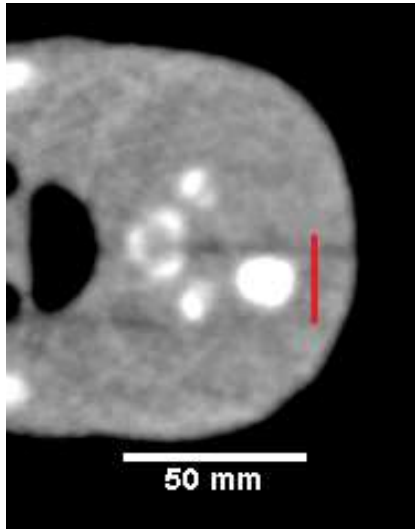

(b)
Fig. 9 Close-up view, with enhanced contrast, of the titanium prosthesis region: (a) x-CT shown in figure 7; (b) pCT shown in figure 6 (a). Red line indicates the region of interest (ROI) used to quantify the standard deviation of the HU and RSP values.

The two ROIs are one pixel wide, $32.3 \mathrm{~mm} \mathrm{long}$, and distant $11.3 \mathrm{~mm}$ and $20.2 \mathrm{~mm}$ respectively from tungsten and titanium prosthesis. They are shown in figures 8 (b) and 9 (b) as red lines.

Figures 10 and 11 show the standard deviation normalized to the average value in both ROIs, as a function of the axial coordinate. In the regions away from the artifacts, the standard deviation in $\mathrm{x}-\mathrm{CT}$ is lower than in pCT images $(\sim 1 \%$ against $\sim 2 \%$ ). In the $\mathrm{pCT}$ one, the standard deviation is higher 
than $0.6 \%$, value obtained in the EDC phantom, probably due to an intrinsic non-uniformity of the head phantom. Nonetheless, in the $\mathrm{x}-\mathrm{CT}$ ROIs close to the tungsten and titanium implants, fluctuations are up to $6 \%$ and $12 \%$, respectively, while these artifacts are reduced to a $2-3 \%$ in pCT images in the same regions.

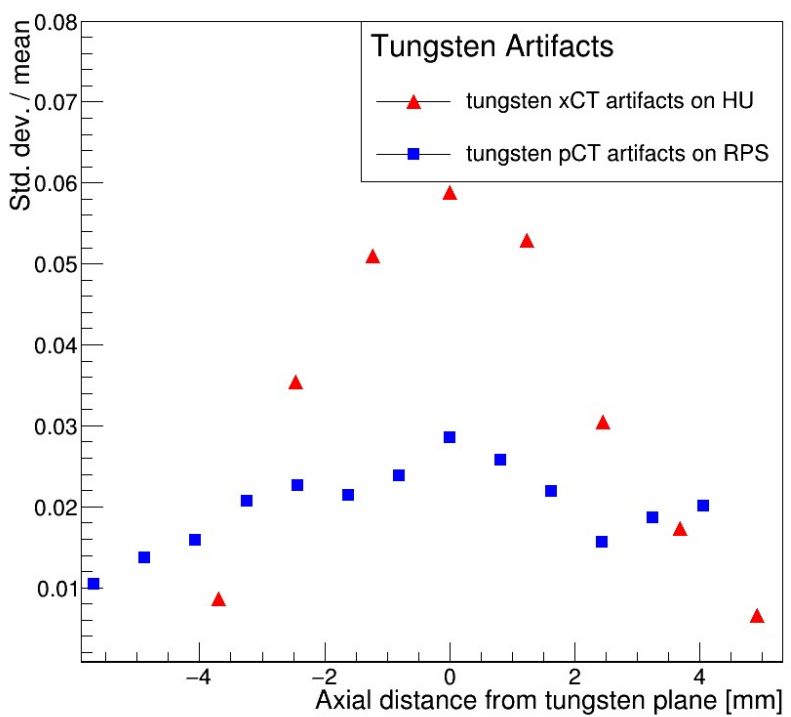

Fig. 10 RSP (pCT blue square) and HU (x-CT red triangle) standard deviation, normalized to the RSP or HU average value, calculated within the ROI close to the tungsten dental filling shown in figure 8 (b). In abscissa, the axial distance means the vertical distance from the plane of figures 6 (a) and 7.

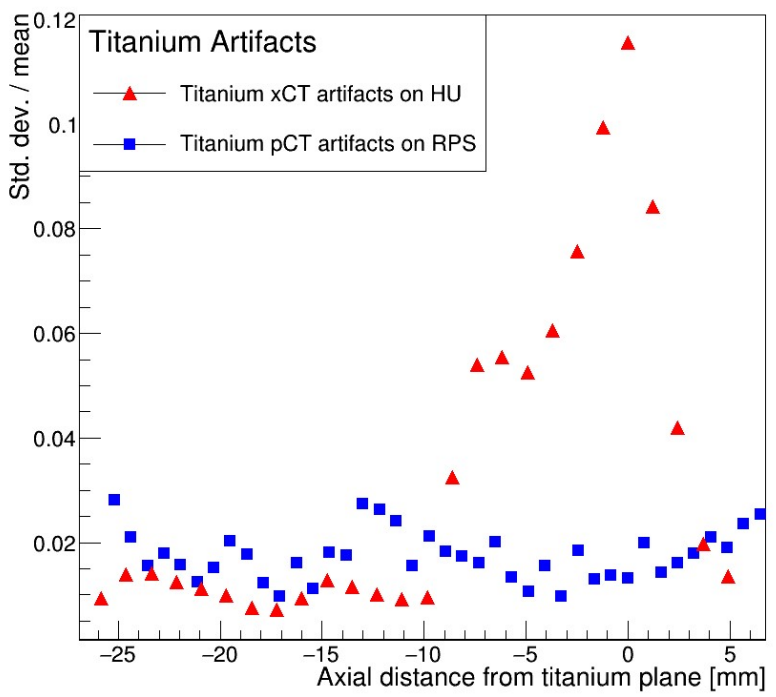

Fig. 11 RSP (pCT blue square) and HU (x-CT red triangle) standard deviation, normalized to the RSP or HU average value, calculated within the ROI close to the titanium prosthesis shown in figure 9 (b). In abscissa, the axial distance means the vertical distance from the plane of figures 6 (a) and 7.

\section{Conclusions}

A proton Computed Tomography ( $\mathrm{pCT}$ ) system, composed by a microstrip silicon tracker and a YAG:Ce calorimeter, has been manufactured and tested at the Trento proton Therapy Center by our collaboration. Measurements have been carried out using a $211 \mathrm{MeV}$ proton beam with a certified electron density calibration phantom. Tomography images obtained by customized iterative algebraic reconstruction algorithms show that our system is able to correlate measured and expected RSP with discrepancies less than $1 \%$. Such a precise direct measurement of Relative Stopping Power maps demonstrates that our pCT system could be indeed quite beneficial for improving quality assurance protocols in particle therapy facilities. Further, a main open issue in hadron therapy is how to handle artifacts in $\mathrm{x}-\mathrm{CT}$ images due to the presence of metal prosthesis. We therefore performed a first quantitative comparison of artifacts produced by metal prosthesis in $\mathrm{x}-\mathrm{CT}$ and pCT. Tests have been carried out with our device in the same proton beam using an anthropomorphous head phantom carrying a titanium spinal bone prosthesis and a tungsten dental filling. Reconstructed pCT images evidence a significant reduction of artifacts in the proximity of prosthesis against those found in $\mathrm{x}-\mathrm{CT}$ ones. Standard deviations normalized to mean values up to $12 \%$ and $6 \%$ have been found for $\mathrm{x}-\mathrm{CT}$ in ROIs distant about $2 \mathrm{~cm}$ from titanium spinal bone and $1 \mathrm{~cm}$ from tungsten dental filling respectively. In same ROIs of pCT images, standard deviations are reduced to about $2-3 \%$. This further experimental result evidences the potential benefit of using $\mathrm{pCT}$ as a tool for improving tumor targeting precision in hadron therapy.

\section{Acknowledgements}

This work has been performed in the framework of PRIMA-RDH-IRPT_PP experiments funded by INFN-CSN5 and MIUR, Italy.

\section{References}

[1] Schneider U, Pedroni E and Lomax A 1996 The calibration of CT Hounsfield units for radiotherapy treatment planning Phys. Med. Biol. 41 111-124, 10.1088/0031-9155/41/1/009.

[2] Paganetti H 2012 Range uncertainties in proton therapy and the role of Monte Carlo simulations Phys. Med. Biol. 57 R99R117, 10.1088/0031-9155/57/11/R99.

[3] Giantsoudi D et al. 2017 Metal Artifacts in Computed Tomography for Radiation Therapy Planning: Dosimetric Effects and Impact of Metal Artifact Reduction Phys. Med. Biol. 62, 10.1088/1361-6560/aa5293.

[4] Johnson R P 2018 Review of medical radiography and tomography with proton beams Rep. Prog. Phys. 81016701 , 10.1088/1361-6633/aa8b1d.

[5] Vanzi E et al. 2013 The PRIMA collaboration: Preliminary results in FBP reconstruction of $\mathrm{pCT}$ data Nucl. Instr. and Meth. A 730 184-190, 10.1016/j.nima.2013.05.193. 
[6] Civinini C et al. 2013 Recent results on the development of a proton computed tomography system $\mathrm{Nucl}$. Instr. and Meth. A 730 573-576, 10.1016/j.nima.2013.05.147.

[7] Scaringella M et al. 2013 The PRIMA (PRoton IMAging) collaboration: Development of a proton Computed Tomography apparatus Nucl. Instr. and Meth. A 730 178-183, 10.1016/j.nima.2013.05.181.

[8] Scaringella M et al. 2014 A proton Computed Tomography based medical imaging system JINST 9 C 12009 , 10.1088/1748-0221/9/12/C12009.

[9] Sipala V et al. 2015 Design and characterisation of a YAG(Ce) calorimeter for proton Computed Tomography application JINST 10 C 03014, 10.1088/1748-0221/10/03/C03014.

[10] Bruzzi M et al. 2017 Proton computed tomography images with algebraic reconstruction Nucl. Instr. and Meth. A 845 652-655, 10.1016/j.nima.2016.05.056.

[11] Civinini C et al. 2017 Proton Computed Tomography: iterative image reconstruction and dose evaluation JINST 12 C01034, 10.1088/1748-0221/12/01/C01034.

[12] Civinini C et al. 2016 Proof-of-Principle results of proton computed tomography in 2016 IEEE Nuclear Science Symposium, Medical Imaging Conference and RoomTemperature Semiconductor Detector Workshop (NSS/MIC/RTSD), Strasbourg, 1-6, 10.1109/NSSMIC.2016.8069620.

[13] Williams D C 2004 The most likely path of an energetic charged particle through a uniform medium Phys. Med. Biol. 49 2899-2911, 10.1088/0031-9155/49/13/010.

[14] Shulte R W, Penfold S N, Tafas J T and Shubert K E 2008 A maximum likelihood proton path formalism for application in proton computed tomography Med. Phys. 35 4849-4856, 10.1118/1.2986139.

[15] Li T, Liang Z, Singanallur J V, Satogata T J, Williams D C and Schulte R W 2006 Reconstruction for proton computed tomography by tracing proton trajectories - A Monte Carlo study Med. Phys. 33 699-706, 10.1118/1.2171507.

[16] Sipala V et al. 2017 A binary readout chip for silicon microstrip detector in proton imaging application JINST 12 C01030, 10.1088/1748-0221/12/01/C01030.

[17] Agostinelli S et al. 2003 GEANT4: A Simulation toolkit Nucl. Instr. and Meth. A 506 250-303, 10.1016/S01689002(03)01368-8.

[18] Allison J et al. 2006 Geant4 Developments and Applications IEEE Transactions on Nuclear Science 53 270-278, 10.1109/TNS.2006.869826.

[19] Allison J et al. 2016 Recent developments in GEANT4 Nucl. Instr. and Meth. A 835 186-225, 10.1016/j.nima.2016.06.125.

[20] Gomà C, Almeida I P and Verhaegen F 2018 Revisiting the single-energy CT calibration for proton therapy treatment planning: a critical look at the stoichiometric method Phys. Med. Biol. 63 235011, 10.1088/1361-6560/aaede5.

[21] Tommasino F et al. 2017 Proton beam characterization in the experimental room of the Trento Proton Therapy facility $\mathrm{Nucl}$. Instr. and Meth. A 869 15-20, 10.1016/j.nima.2017.06.017.

[22] Gordon R, Bender R and Herman G T 1970 Algebraic reconstruction techniques (ART) for three-dimensional electron microscopy and x-ray photography J. Theor. Biol. 29 471-81, 10.1016/0022-5193(70)90109-8.
[23] Berger M J, Coursey J S, Zucker M A and Chang J 2017 Stopping-Power \& Range Tables for Electrons, Protons, and Helium Ions NIST Standard Reference Database 124, 10.18434/T4NC7P.

[24] Wang D, Mackie T R and Tomé W A 2010 On the use of a proton path probability map for proton computed tomography reconstruction Med.Phys. 37 4138-4145, 10.1118/1.3453767.

[25] Andersen A H and Kak A C 1984 Simultaneous Algebraic Reconstruction Technique (SART): A superior implementation of the ART algorithm Ultrasonic Imaging $\mathbf{6}$ 8-94, 10.1016/0161-7346(84)90008-7.

[26] Censor Y, Elfving T, Herman G T and Nikazad T 2008 On Diagonally Relaxed Orthogonal Projection Methods SIAM Journal on Scientific Computing 30 473-504, $10.1137 / 050639399$.

[27] Rueden C T et al. 2017 ImageJ2: ImageJ for the next generation of scientific image data BMC Bioinformatics 18 529, 10.1186/s12859-017-1934-z. 